Journal of Clinical and Nursing Research

Research Article

\title{
The Analysis of Clinical Features of Systemic Lupus Erythematosus (SLE) in Children
}

Ying Liang, Pingping Zhang, Huiqin Chen, Xiangqin Luo, Yesheng Ling, Yating Li, Ou Jin, Qian Kong, Fenhua Chen, Yikun Mou*

Guangzhou City of Guangdong Province, the Third Affiliated Hospital of Sun Yat-sen University, Guanghzou 510630, China

[Abstract] Objective: Observe the clinical characteristics of children with SLE, namely, to observe the symptoms and laboratory examinations, such as blood routine, blood lipid, immunoglobulin, complement, autoantibodies, serum $25(\mathrm{OH}) \mathrm{D}$ and other indicators, and to explore the clinical characteristics, the difference and the significance of vitamin D supplements between male and female SLE patients in children respectively. Methods: We enrolled 64 cases of SLE patients in children who were admitted into the department of pediatrics and rheumatology of the third affiliated hospital of sun yat-sen university in guangzhou from May 1, 2011 to February 1, 2019, They were analyzed retrospectively, adopting $\mathrm{X}^{2}$ test for statistical analysis. Results: 64 cases of SLE in children, which included 10 cases of male and 54 cases of female. Clinical manifestations: facial skin rash in 48 patients $(75 \%)$, fever in 38 cases (59.4\%), arthritis in 28 cases (43.8\%), oral ulcer in 18 cases $(28.1 \%)$, serositis in 13 cases $(20.3 \%)$, and the sun allergy in 9 cases $(14.1 \%)$, the damage of central nervous system in 7 cases $(10.9 \%)$. Laboratory examination: 30 cases of leukopenia (46.9\%), anemia in 30 cases $(46.9 \%)$, thrombocytopenia in 12 cases $(18.8 \%)$, hematuria in 18 cases $(28.1 \%)$, proteinuria in 33 cases $(51.2 \%), 6$ patients with renal impairment $(9.4 \%)$, antinuclear antibody positive in 63 cases (98.4\%), anti-double-stranded DNA (dsDNA) antibody positive in 48 cases $(75 \%)$, anti SSA antibody positive in 44 cases (68.7\%), SSB antibody positive in 33 cases $(51.6 \%)$, $\mathrm{Sm}$ antibody positive in 40 cases $(62.5 \%)$, nucleosome antibody positive in 28 cases $(43.8 \%)$. Among these children, male SLE patients were higher than female children with SLE in the damage of kidney, Sm anti- bodies and resisting nucleosome antibody positive rates $\left(\mathrm{X}^{2}=4.451,8.336,6.803, \mathrm{P}<0.05\right)$, the female children with SLE was higher than male SLE Children in the anti-SSB antibody positive rate $\left(\mathrm{X}^{2}=4.945, \mathrm{P}<0.05\right)$. In 64 cases of SLE children, which included 52 cases were lower than the normal level of serum $25(\mathrm{OH}) \mathrm{D}$ measurements, 12 cases were in the normal lower limit of serum $25(\mathrm{OH}) \mathrm{D}$ measurements, at the same time, the female SLE. Patients was higher than male children with SLE in the reduce rate of serum $25(\mathrm{OH}) \mathrm{D}\left(\mathrm{X}^{2}=\right.$ 8.351, $\mathrm{P}<0.05$ ). Conclusion: Male SLE patients which appeared damage of kidney easier than female patients, the proteinuria was the most common in the damage of kidney. Resistance to Sm antibodies which was the risk factor of renal injury with higher incidence in male children with SLE; Anti nucleosome antibody which was the risk factor for the disease activity in male children with SLE were higher than female children with SLE. It was estimated that the risk of Sjogren's syndrome appeared in female with SLE were higher than that in male SLE children. In this retrospective study, the serum 25 $(\mathrm{OH})$ D levels were significantly lower in children with SLE, and vitamin D supplementation was required.

Key words: Children; Systemic lupus erythematosus(SLE); Clinical features; 25(OH)D

Publication date: September, 2020

Publication online: 30 September, 2020

*Corresponding author: Yikun Mou, liangying0906@126.com

\section{Introduction}

Systemic lupus erythematosus (SLE) is a chronic au- 
toimmune diseases which affected multi system, multi organ involvement and caused immune dysfunction, the incidence of female patients were significantly higher than male, the clinical manifestations varied and duration complicated. The etiology is still not completely clear, generally regarded as with the environment, genetic and sex hormones. In order to investigate the differences of clinical features and different gender children SLE children, the study enrolled 64 cases of SLE patients in children who were admitted into the department of pediatrics and rheumatology of the third affiliated hospital of sun yat-sen university in guangzhou from May 1, 2011 to February 1, 2019. The datas were analyzed retrospectively and summarized as follows.

\section{Materials and methods}

\subsection{Clinical data}

Laboratory related clinical data and of 64 cases SLE children which all newly diagnosed and collected in the third affiliated hospital of sun yat-sen university from May 1, 2011 to February 1, 2019, and collected the serum $25(\mathrm{OH}) \mathrm{D}$ index during periodic review, all groups with the length of data are completely. The diagnosis is in line with the 2009 SLE international clinical cooperation group (SLICC) SLE standard.

\subsection{Observation items}

Record the clinical symptoms such as fever, facial rash, sun allergies, arthritis, alopecia, oral ulcer, serositis (pleurisy and / or pericarditis), kidney damage (hematuria, proteinuria, blood urea nitrogen and / or creatinine increased), nervous system damage (visual impairment, psychiatric symptoms, epileptic symptoms, cranial nerve damage, encephalosis). The same day or next day after admission completed the blood routine test, biochemical test (fasting), immunoglobulin, complement (Ig), antinuclear antibody, ENA spectrum, and improved of serum $25(\mathrm{OH})$ D levels timely, selected the first test indicators included in the comparison of the study. Record the blood system damage (leukopenia $<4 * 10^{\wedge}$ 9/L, hemoglobin $<120 \mathrm{~g} / \mathrm{L}$, thrombocytopenia and $<100^{*} 10^{\wedge} 9 / \mathrm{L}$ ) and the following abnormalities (such as $\mathrm{IgG}>16 \mathrm{~g} / \mathrm{L}$, high density lipoprotein $<0.78 \mathrm{mmol} /$ $\mathrm{L}$, low density lipoprotein $>3.10 \mathrm{mmol} / \mathrm{L}, \mathrm{C} 3<850 \mathrm{mg} /$ $\mathrm{L}, \mathrm{C} 4<120 \mathrm{mg} / \mathrm{L}$, anti nuclear antibody (ANA $\geq 1: 160$ ), anti double stranded DNA (dsDNA) antibody $\geq 30 \mathrm{IU} /$ $\mathrm{ml}$,anti Sm antibody, anti SSA antibody, anti SSB antibody, antinuclear antibody positive and serum $25(\mathrm{OH})$
$\mathrm{D}[25(\mathrm{OH}) \mathrm{D}<49.7 \mathrm{nmol} / \mathrm{L}]$.

\subsection{Statistical processing}

Using SPSS 19.0 statistical software for statistical analysis, normal distribution of measurement data to $(\overline{\mathrm{x}} \pm \mathrm{s})$ said, enumeration data using $\mathrm{X}^{2}$ test, $\mathrm{P}<0.05$ said the difference was statistically significant.

\section{Result}

\subsection{In general}

64 cases of SLE in children,which included 10 cases of male and 54 cases of female. the ratio of male to female was 1.0: 5.4 , age $(10.14 \pm 3.06)$ years old, the peak age of onset was 9-15 years old.

2.2 The clinical manifestations

(1) SLE patients with clinical symptoms varied, the first symptom of 64 cases in children were as follows: facial rash in 48 cases $(75 \%)$, fever in 38 cases $(59.4 \%), 28$ cases of arthritis ( $43.8 \%), 18$ cases of oral ulcer (28.1\%), 13 patients with serositis $(20.3 \%)$, sun allergy in 9 cases $(14.1 \%), 7$ cases of central nervous system damage (10.9\%), the most common initial symptoms of visible was facial rash. Laboratory examination revealed: 36 cases of blood system damage $(56.3 \%)$ [30 cases of leukopenia (46.9\%), 30 cases of anemia( $46.9 \%)$, thrombocytopenia in 12 cases (18.8\%)], 35 cases of kidney damage $(54.7 \%)$ [ 18 cases of hematuria $(28.1 \%)$, proteinuria in 33 cases $(51.2 \%)$, renal damage (creatinine and / or urea nitrogen increased ) in 6 cases (9.4\%)], antinuclear antibody positive in 63 cases $(98.4 \%)$, anti double stranded DNA (dsDNA) antibody positive in 48 cases $(75 \%)$, anti SSA antibody positive in 44 cases $(68.7 \%)$, Anti SSB antibody positive in 33 cases (51.6\%), anti Sm antibody positive in 40 cases $(62.5 \%)$, anti nucleosome antibody positive in 28 cases (43.8\%), serum $25(\mathrm{OH}) \mathrm{D}$ which were lower than the normal low limit value in 52 cases(81.3\%). (2)Male SLE patients were prone to kidney damage, proteinuria was the most common, the renal injury incidence was significantly higher than that of female patients $\left(\mathrm{X}^{2}=4.451\right.$, $\mathrm{P}=0.039$ ); male patients with positive anti $\mathrm{Sm}$ antibody was higher than that of female patients $\left(\mathrm{X}^{2}=8.336\right.$, $\mathrm{P}=0.005)$; the positive rate of male patients with SLE anti nucleosome antibody was higher than female children $\left(\mathrm{X}^{2}=6.803, \mathrm{P}=0.011\right)$; the positive rate of female children with anti SSB antibody was higher than that of male children $\left(\mathrm{X}^{2}=4.945, \mathrm{P}=0.030\right)$. The mean of serum $25(\mathrm{OH}) \mathrm{D}$ in 64 cases was $28.16 \mathrm{nmol} / \mathrm{L}$, includ- 
ing 52 cases with serum $25(\mathrm{OH}) \mathrm{D}$ were lower than the normal level, and 1 cases of female children with $25(\mathrm{OH}) \mathrm{D}$ minimum value was only $14.3 \mathrm{nmol} / \mathrm{L}$; the female SLE patients was higher than male children with SLE in the reduce rate of serum $25(\mathrm{OH}) \mathrm{D}\left(\mathrm{X}^{2}=8.351\right.$, $\mathrm{P}<0.05$ ); female SLE was higher than male patients with SLE in anemia incidence $\left(\mathrm{X}^{2}=6.975, \mathrm{P}=0.01\right)$. The rest of the facial skin rash, fever, arthritis, oral ulcer, sun allergy, serositis, neutropenia and thrombocytopenia, nervous system damage, the rise of IgG, high density lipoprotein decreased ,low density lipoprotein increased, complement $\mathrm{C} 3$ decreased, complement $\mathrm{C} 4$ decreased, ANA positive, anti dsDNA antibody positive, anti SSA positive were not significant difference between male and female children. The chart below reflect the specific circumstances. Show in Table 1.

\section{Discussion}

Systemic lupus erythematosus (SLE) is a chronic autoimmune disease characterized by multiple system, multiple organ involvement and multiple autoantibodies ${ }^{[1]}$. The main clinical manifestations were skin mucosa, arthritis, kidney damage, blood system damage, serositis, damage to the central nervous system and other organs. The disease is common in women of adult, childhood onset rate is about $15 \%-20 \%{ }^{[2]}$. In this study, the ratio of male to female was 1:5.4, which was similar to the literature, suggesting that SLE in children had the obvious tendency of gender ${ }^{[3]}$.

The clinical symptoms of children with SLE were more severe than that of adult patients, often involving blood, kidney, heart, central nervous system and so

Table 1. Clinical and serological examination of male and female patients with SLE (case number (n\%)

\begin{tabular}{|c|c|c|c|c|c|}
\hline Project & Total (64 cases) & Male (10cases) & Female (54cases) & $\mathrm{X}^{2}$ & $\mathrm{P}$ \\
\hline Facial rash & $48(75.0 \%)$ & $6(60 \%)$ & $42(77.8 \%)$ & 1.409 & 0.240 \\
\hline Fever & $38(59.4 \%)$ & $4(40 \%)$ & $34(63.0 \%)$ & 1.84 & 0.180 \\
\hline Oral ulcer & $18(28.1 \%)$ & $3(30 \%)$ & $15(27.8 \%)$ & 0.020 & 0.890 \\
\hline Sun allergy & $9(14.1 \%)$ & $1(10 \%)$ & $8(14.8 \%)$ & 0.157 & 0.693 \\
\hline Arthritis & $28(43.8 \%)$ & $2(20 \%)$ & $26(48.1 \%)$ & 2.748 & 0.102 \\
\hline Serositis & $13(20.3 \%)$ & $2(20 \%)$ & $11(20.4 \%)$ & 0.001 & 0.979 \\
\hline Kidney damage & $35(54.7 \%)$ & $10(10 \%)$ & $25(46.3 \%)$ & 4.451 & 0.039 \\
\hline proteinuria & $33(51.2 \%)$ & $9(90 \%)$ & $24(44.4 \%)$ & 7.627 & 0.008 \\
\hline hematuria, & $18(28.1 \%)$ & $4(40 \%)$ & $14(25.9 \%)$ & 0.811 & 0.371 \\
\hline urea nitrogen and / or creatinine increased & $6(9.4 \%)$ & $3(30 \%)$ & $3(5.6 \%)$ & 6.336 & 0.014 \\
\hline blood system damage & $36(56.3 \%)$ & $4(40 \%)$ & $32(59.3 \%)$ & 1.257 & 0.267 \\
\hline leukopenia & $30(46.9 \%)$ & $4(40 \%)$ & $26(48.1 \%)$ & 0.219 & 0.642 \\
\hline anemia & $30(46.9 \%)$ & $1(10 \%)$ & $29(53.7 \%)$ & 6.975 & 0.010 \\
\hline thrombocytopenia & $12(18.8 \%)$ & $2(20 \%)$ & $10(18.5 \%)$ & 0.012 & 0.914 \\
\hline nervous system damage & $7(10.9 \%)$ & $1(10 \%)$ & $6(11.1 \%)$ & 0.010 & 0.919 \\
\hline IgG increased & $46(71.9 \%)$ & $6(60 \%)$ & $40(74.1 \%)$ & 0.811 & 0.371 \\
\hline C3 decreased & $56(87.5 \%)$ & $8(80 \%)$ & $48(88.9 \%)$ & 0.596 & 0.443 \\
\hline C4 decreased & $42(65.6 \%)$ & $6(60 \%)$ & $36(66.7 \%)$ & 0.161 & 0.689 \\
\hline HDL decreased & $27(42.2 \%)$ & $4(40 \%)$ & $23(42.6 \%)$ & 0.023 & 0.881 \\
\hline LDL increased & $18(28.1 \%)$ & $3(30 \%)$ & $15(27.8 \%)$ & 0.020 & 0.888 \\
\hline ANA positive & $63(98.4 \%)$ & $10(100 \%)$ & $53(98.1 \%)$ & 0.183 & 0.670 \\
\hline anti Sm antibody positive & $40(62.5 \%)$ & $9(90 \%)$ & $31(57.4 \%)$ & 8.336 & 0.005 \\
\hline anti SSA antibody positive & $44(68.7 \%)$ & $7(70 \%)$ & $37(68.5 \%)$ & 0.008 & 0.927 \\
\hline anti SSB antibody positive & $33(51.6 \%)$ & $2(20 \%)$ & $31(57.4 \%)$ & 4.945 & 0.03 \\
\hline Anti nucleosome antibody positive & $28(43.8 \%)$ & $8(80 \%)$ & $20(37.0 \%)$ & 6.803 & 0.011 \\
\hline Serum $25(\mathrm{OH}) \mathrm{D}$ decreased & $52(81.3 \%)$ & $6(60 \%)$ & $46(85.2 \%)$ & 8.351 & 0.005 \\
\hline
\end{tabular}


on $[4,5]$.The eastern city of China-Shanghai also reported a higher involvement rate of kidney, blood system and respiratory system in children with SLE. This study were mainly from the southern region of China, the most common clinical symptoms in children were facial rash, followed by fever, then followed by blood system damage, kidney damage was also very common. The most common laboratory test was antinuclear antibody positive and complement $\mathrm{C} 3$ decreased, which the incidence were $>85 \%$; followed by serum $25(\mathrm{OH})$ D decreased, the incidence was $>80 \%$; anti double stranded DNA antibody, anti SSA antibody positive, IgG increased, C4 complement decreased ,which the incidence were $>65 \%$. anti $\mathrm{Sm}$ antibodies, anti SSB antibody positive rates were $>50 \%$; In addition, anti nucleosome antibodies and high density lipoprotein increased were also common, the rates were $>40 \%$.

Another study in Shanghai of China, showed that renal involvement was more common in male with SLE, and with rapid progression and poor prognosis 。 This study also found that male SLE patients were significantly higher than female children with SLE in the damage of kidney, the most common was proteinuria, then hematuria, the last was renal damage. Therefore, the earlier diagnosis and clear the biopsy pathological types of renal tissue and aggressive treatment, the more significance to the male SLE patients.

The rate of anti Sm antibody positive on the 40/64 (62.5\%).Mariangel Arroyo- Vila et al. in 2322 cases of different races (African American, ethnicity defined Hispanic or Caucasian)) study of SLE patients with an average age of 34.4 , the positive rate of anti $\mathrm{Sm}$ antibody in $24.9 \%$, anti-Sm antibodies were associated with several clinical features including serious manifestations such as renal, neurologic, and hematologic disorders as well as vasculitis ${ }^{[6]}$. Anti Sm antibody was also a landmark antibody in children with SLE, which was great significance in the clinical diagnosis of $\mathrm{SLE}^{[7]}$. This study showed that the antibody positive rate of children SLE patients were higher than that of the adult patients reported by Mariangel ArroyoVila et al. the positive rate in male patients with anti Sm antibody was significantly higher than that of female, and male children with renal involvement in children was significantly higher than that of female patients, which explained anti Sm antibody may be sensitive biomarkers of renal injury in male SLE patients .Therefore, for male SLE patients in the clinical work period, especially in male patients with positive anti Sm antibody, even though it does not appear the damage of kidney, later also need to regularly visit, pay attention to urine, renal function and other aspects of the examination, if the abnormal index appear, performed renal biopsy timely ,then to early detection of the renal damage, and reduce misdiagnosis. The pathogenesis and genetic factors related to SLE, DR2/DR3 heterozygous genotype HLA is associated with at least one antibody to soluble nuclear antigen, DR3/DR3 genotype and the anti Sm antibody are strongly correlated, the study of spontaneous lupus NZM2328.DR3+AE0 transgenic mice showed that HLA-DR3 molecules play a key role in the pathogenesis of SmD antigen of autoimmune reaction and of lupus nephritis ${ }^{[8]}$.

Clinical studies had demonstrated that the anti nucleosome antibody as a diagnostic index of SLE wit high sensitivity and specificity, and associated with the disease activity ${ }^{[9]}$. It had been reported better than that of complement $\mathrm{C} 3$ and anti ds-DNA antibody in the forecast of long-term activity of SLE ${ }^{[10]}$. It had been reported male children with SLE had higher disease activity than female children. This study showed that the positive rate of anti nucleosome antibodies in male children with SLE was significantly higher than that of female children, suggesting that anti nucleosome antibody positive was one of sensitive indicators to reflect the male children with SLE disease activity. So in clinical work period, anti nucleosome antibodies in children with SLE should be used as a routine examination of disease activity monitoring especially in male children.

SLE and Sjogren syndrome (SS) are the common autoimmune disease. Clinically, Sjogren's syndrome can exist alone, also can be secondary to SLE or coexist with it. Anti SSB antibody is an important diagnostic basis SLE and $\mathrm{SS}^{[1]}$. Studies had found that anti SSB antibody positive rate in SLE secondary SS were higher than pure SLE patients。The study found that anti SSB positive rate in female with SLE children was significantly higher than male patients, female patients with SLE showed that the risk of future in SS were higher than that of male children. So for female with SLE, monitoring of anti SSB antibody regularly, early recognition of SLE secondary to SS, so as to achieve early discovery, early diagnosis, early treatment, and then do the development to prevent or slow the disease.

The concentration of $25(\mathrm{OH}) \mathrm{D}$ in the circulation is stable, serum $25(\mathrm{OH})$ D levels can reflect the nutrition- 
al status of vitamin D in the body. Vitamin D (VitD) is to maintain bone metabolism, regulate calcium and phosphorus metabolism of fat soluble steroid derivatives, it is an important organ in bone nutrition and one of the important drugs prevention and treatment of osteoporosis. Vitamin D3 is stored in the body form of vitamin $\mathrm{D}$, the main loop for its form was generated by the liver into $25(\mathrm{OH}) \mathrm{D}$, the latter by chemical conversion of the kidney, forming a highly active 1, 25 $(\mathrm{OH}) 2 \mathrm{D}$, which play biological effects after binding to the vitamin D receptor. In this paper, 64 cases of SLE patients with serum $(\mathrm{OH}) \mathrm{D}$ determination, found that there were 52 cases of serum SLE $(\mathrm{OH})$ D levels were significantly reduced, and the reduction rate of female children was significantly higher than that of male children patients, Analysis of the causes related to the following factors: SLE patients with photosensitivity, the skin is lack of sunlight then induced skin to generate endogenous vitamin D decreased; SLE occurs in women, while SLE can cause the hypothalamic pituitary ovarian axis dysfunction, estrogen levels decline due to the levels of vitamin D decreased; Causes of long-term hormone therapy: SLE patients need long-term glucocorticoid treatment, fracture caused by osteoporosis is one of the serious adverse reactions to glucocorticoid, it related to 1- $\alpha$ hydroxylase activity decline from the patients with SLE renal damage and long-term hormone therapy lead to vitamin D metabolism disorders. Kamen et $\mathrm{al}^{[12]}$ Reported that the vitamin $\mathrm{D}$ levels in patients with SLE compared with healthy controls, SLE patients with vitamin D deficiency of $67 \%$; Ruiz-Irastorza et al. reported that, in 92 cases of SLE female patients, $90 \%$ of patients with serum $25(\mathrm{OH})$ D deficiency; The SLE disease and drug treatment to patients with liver, kidney and intestinal function decreased, resulting in the absorption of vitamin D, synthesis, biological conversion disorder in patients .SLE patients lack of vitamin D can lead to reduced intestinal absorption of calcium, calcium and phosphorus metabolism, resulting in more bone loss, earlier occurrence of osteoporosis. In 2008 the National Osteoporosis Foundation (NOF) announced the latest "American osteoporosis physician guide" suggested that the premise for any anti osteoporosis drug application should have adequate calcium and vitamin D. In addition, studies in recent years have confirmed that the biological active form of vitamin $\mathrm{D}-1,25(\mathrm{OH}$ 2D , which is a kind of multifunctional steroids, participated in a variety of cell proliferation, differentiation and regulation of immune function, vitamin $\mathrm{D}$ deficien- cy can cause the immune function of patients with hyperthyroidism, and involve in the pathogenesis of SLE. Doria et $a l^{[14]}$ studies suggest that vitamin $\mathrm{D}$ is a potential immunoregulatory factor, can improve the research condition of SLE and treated SLE. Kamen et $a l^{[13]}$ study suggest that the content of vitamin D decreased is the risk factors of SLE. In this study,81.3\% (52/64) patients were significantly lower in $25(\mathrm{OH}) \mathrm{D}$ levels, indicated the metabolic disorder of $25(\mathrm{OH}) \mathrm{D}$, it was in accordance with the above research.. So in the treatment of SLE with vitamin D is not only safe but also has great significance in clinical treatment. In clinical, give oral calcium supplementation and Calcitriol Soft Capsules to the patients, have been used for SLE and other immune related diseases, and achieved good results.

In summary, clinical workers for SLE children with different gender, attention should be focused on some emphasis. The related indexes of male SLE patients, should pay attention to kidney damage (urine routine, renal function, renal biopsy), the monitoring of anti Sm antibody and anti nucleosome antibody; female SLE with anti SSB antibody positive should be paid more attention. Moreover, all the SLE children should be paid attention to regular review of serum $25(\mathrm{OH}) \mathrm{D}$, and the importance of activity of vitamin D supplement, so as to better diagnosis and control of diseases, and delay the progress.

\section{References}

[1] Harvey, P.R. and C. Gordon, B-cell targeted therapies in systemic lupus erythematosus: successes and challenges. BioDrugs, 2013. 27(2): 85-95.

[2] Hiraki, L.T., et al., Clinical and laboratory characteristics and long-term outcome of pediatric systemic lupus erythematosus: a longitudinal study. J Pediatr, 2008. 152(4): 550-6.

[3] Bader-Meunier, B., et al., Initial presentation of childhood-onset systemic lupus erythematosus: a French multicenter study. J Pediatr, 2005. 146(5): 648-53.

[4] Gokce, M., et al., Hematological features of pediatric systemic lupus erythematosus: suggesting management strategies in children. Lupus, 2012. 21(8): 878-84.

[5] Amaral, B., et al., A comparison of the outcome of adolescent and adult-onset systemic lupus erythematosus. Rheumatology (Oxford), 2014. 53(6): 1130-5.

[6] Arroyo-Avila, M., et al., Clinical associations of anti-Smith antibodies in PROFILE: a multi-ethnic lupus cohort. Clin Rheumatol, 2015. 34(7): 1217-23.

[7] Mahler, M., Sm peptides in differentiation of autoimmune diseases. Adv Clin Chem, 2011. 54: 109-28.

[8] Chowdhary, V.R., et al., A Central Role for HLA-DR3 in Anti-Smith Antibody Responses and Glomerulonephritis in a Transgenic Mouse Model of Spontaneous Lupus. J Immunol, 
2015. 195(10): 4660-7.

[9] Gutierrez-Adrianzen, O.A., et al., Diagnostic value of anti-nucleosome antibodies in the assessment of disease activity of systemic lupus erythematosus: a prospective study comparing anti-nucleosome with anti-dsDNA antibodies. J Rheumatol, 2006. 33(8): 1538-44.

[10] Li, T., et al., Anti-nucleosome antibodies outperform traditional biomarkers as longitudinal indicators of disease activity in systemic lupus erythematosus. Rheumatology (Oxford), 2015.
54(3): 449-57.

[11] Franceschini, F. and I. Cavazzana, Anti-Ro/SSA and La/SSB antibodies. Autoimmunity, 2005. 38(1): 55-63.

[12] Kamen, D.L., et al., Vitamin D deficiency in systemic lupus erythematosus. Autoimmun Rev, 2006. 5(2): 114-7.

[13] Ruiz-Irastorza, G., et al., Vitamin D deficiency in systemic lupus erythematosus: prevalence, predictors and clinical consequences. Rheumatology (Oxford), 2008. 47(6): 920-3. 